\title{
The uprising of metabolic dysfunction-associated fatty liver disease (MAFLD) in acute-on-chronic liver failure (ACLF)
}

\author{
Kenneth I. Zheng ${ }^{1}$, Ming-Hua Zheng ${ }^{1,2,3}$ \\ ${ }^{1}$ NAFLD Research Center, Department of Hepatology, The First Affiliated Hospital of Wenzhou Medical University, Wenzhou, China; ${ }^{2}$ Institute of \\ Hepatology, Wenzhou Medical University, Wenzhou, China; ${ }^{3}$ Key Laboratory of Diagnosis and Treatment for the Development of Chronic Liver \\ Disease in Zhejiang Province, Wenzhou, China \\ Correspondence to: Ming-Hua Zheng, MD, PhD. NAFLD Research Center, Department of Hepatology, The First Affiliated Hospital of Wenzhou \\ Medical University, No. 2 Fuxue Lane, Wenzhou 325000, China. Email: zhengmh@wmu.edu.cn.
}

Submitted Oct 17, 2021. Accepted for publication Oct 27, 2021.

doi: $10.21037 / \mathrm{hbsn}-21-431$

View this article at: https://dx.doi.org/10.21037/hbsn-21-431

Metabolic dysfunction-associated fatty liver disease (MAFLD) (1) is currently the leading cause of chronic liver disease in the United States (US) and Europe, and the second leading cause for liver transplantation (2). Despite advancements in knowledge over the past decade, MAFLDrelated cirrhosis is the most rapidly growing etiology for acute-on-chronic liver failure (ACLF) in the US (3-5). ACLF is a syndrome characterized by acute deterioration of cirrhosis and extrahepatic failure of 1 or more organs. ACLF represents a significant healthcare burden, and is associated with high short-term mortality. Recently, an epidemiology study showed a 3-fold increase in MAFLDrelated ACLF waitlisted patients in the United Network for Organ Sharing (UNOS) database from 2005 to 2017 (4), and a similar trend was observed in the European Liver Transplant Registry (ELTR) database (5). This is particularly concerning, as the quick rise in MAFLDrelated ACLF has created a major gap between the number of patients requiring a liver transplant and the number of available liver donors.

\section{Historically low awareness of MAFLD}

Before the newly proposed MAFLD terminology was introduced, the term "nonalcoholic fatty liver disease" was used. Over the past 40 years, the diagnosis of nonalcoholic fatty liver disease has evolved to describe hepatic steatosis without excess alcohol ingestion, steatogenic drivers (e.g., the long-term use of steatogenic medication), coeliac disease, lysosomal acid lipase deficiency, or other causes of liver damage, such as viral hepatitis (6). The need to deliberately rule-out competing causes can hamper the diagnostic process. Further, the use of exclusionary criteria to describe a heterogenous disease with multifactorial disease drivers and complex pathophysiology does not translate well to clinical practice (7). Thus, it is not surprising that is a lack of comprehensive data on MAFLDrelated ACLF. This is attributable to past difficulties related to diagnosis and an inadequate therapeutic strategy for the disease. The real-world burden of ACLF due to MAFLD is likely underestimated due to a lack of available data, and large proportion of MAFLD cirrhosis likely remains unclassified (8). The costs and resources required for healthcare continues to grow for these individuals. Thus, more population-based investigations and prospective data may be helpful.

\section{Toward a better classification of MAFLD cirrhosis}

The absence of specific ICD-09 codes for MAFLD cirrhosis complicates advancements in clinical research. In a recent population study of the Healthcare Cost and Utilization Project NIS database, Axley et al. (3). defined etiological MAFLD in ACLF based on obesity absent other known causes of cirrhosis. Similarly, in the UNOS study (4) and ELTR study (5), investigators retrospectively identified MAFLD-ACLF by steatohepatitis-related cirrhosis, or cryptogenic cirrhosis with existing metabolic risk factors, such diabetes and obesity. However, debates continue as to 
whether the terms MAFLD and cryptogenic cirrhosis can be used interchangeably, as a group of individuals $(<5 \%)$ have been identified who match the clinical profile of MAFLD cirrhosis but do not have significant liver steatosis.

Patients with MAFLD may have hepatic fat loss or "burn-out" cirrhosis (9); however, Thuluvath et al. (10) showed that cryptogenic cirrhosis can be the result of MAFLD, immune-mediated hepatitis, or alcoholic liver disease. This suggests cryptogenic cirrhosis can etiologically be MAFLD, but that the 2 terms are not interchangeable. The MAFLD definition by Eslam et al. (11). includes the following inclusionary criteria: prior evidence of live steatosis in combination with past or present metabolic risk factors. The criteria may help streamline the process of diagnosis by identifying past metabolic risk factors as the primary disease modifier by which other liver conditions (e.g., dual etiological viral hepatitis, alcoholic liver injury, and autoimmune disease) may be included due to their contributory role in cirrhosis. Thus, correctly diagnosing cryptogenic or "burn-out" cirrhosis as MAFLD cirrhosis signifies a step in the right direction.

\section{How to address MAFLD-related ACLF}

MAFLD-related ACLF patients had the lowest in-hospital mortality rate compared to those with other etiologies (3); however, as MAFLD-related ACLF transplant registrants are generally older (i.e., aged >60 years), MAFLDrelated ACLF patients have the highest risk of waitlist mortality because they have an increased risk of mortality due to their age $(4,5)$. In addition, metabolic risk factors might predispose patients to system inflammation from adipokines. In patients with alcoholic hepatitis, metabolic risk factors (e.g., obesity and dyslipidemia) increase the severity of alcohol-associated ACLF, and increase the shortterm mortality of these patients (12). Thus, it is conceivable that MAFLD cirrhosis patients with dual etiologies involving alcoholic liver disease might be at greater risk of decompensation, which would further increase the possibility of a precipitating event for ACLF. Improved understandings of the effects of metabolic dysregulation in MAFLD-ACLF patients may lead to improvements in individualized prediction outcomes.

The recent PREDICT study longitudinally investigated 1,273 patients with acute decompensation and identified 4 major precipitants of ACLF that may occur either alone or in combination (13). Among them, bacterial infection and severe alcohol hepatitis were observed in $97 \%$ of the 202 cirrhotic patients who had developed ACLF, while mirrored by the activation of systemic inflammatory affecting many cytokines systems. Liver disease etiology may influence the clinical course of ACLF; however, precipitants of ACLF are etiology independent. Thus, early intervention is important in the treatment of MAFLD cirrhosis. With improved diagnostic criteria and an increased awareness of the disease, clinicians and researchers are undoubtedly better equipped to address the rising health burden associated with MAFLD in ACLF.

\section{Acknowledgments}

Funding: This work was supported by a grant from the National Natural Science Foundation of China (82070588), the High Level Creative Talent Grant from the Department of Public Health in Zhejiang Province (S2032102600032), and a grant from the Project of New Century 551 Talent Nurturing in Wenzhou.

\section{Footnote}

Provenance and Peer Review: This article was commissioned by the editorial office, Hepatobiliary Surgery and Nutrition. The article did not undergo external peer review.

Conflicts of Interest: Both authors have completed the ICMJE uniform disclosure form (available at https://hbsn.amegroups. com/article/view/10.21037/hbsn-21-431/coif). Dr. MHZ serves as the unpaid editorial board member of Hepatobiliary Surgery and Nutrition. The other author has no conflicts of interest to declare.

Ethical Statement: The authors are accountable for all aspects of the work in ensuring that questions related to the accuracy or integrity of any part of the work are appropriately investigated and resolved.

Open Access Statement: This is an Open Access article distributed in accordance with the Creative Commons Attribution-NonCommercial-NoDerivs 4.0 International License (CC BY-NC-ND 4.0), which permits the noncommercial replication and distribution of the article with the strict proviso that no changes or edits are made and the original work is properly cited (including links to both the formal publication through the relevant DOI and the license). See: https://creativecommons.org/licenses/by-nc-nd/4.0/. 


\section{References}

1. Eslam M, Newsome PN, Sarin SK, et al. A new definition for metabolic dysfunction-associated fatty liver disease: An international expert consensus statement. J Hepatol 2020;73:202-9.

2. Wong RJ, Aguilar M, Cheung R, et al. Nonalcoholic steatohepatitis is the second leading etiology of liver disease among adults awaiting liver transplantation in the United States. Gastroenterology 2015;148:547-55.

3. Axley P, Ahmed Z, Arora S, et al. NASH Is the Most Rapidly Growing Etiology for Acute-on-Chronic Liver Failure-Related Hospitalization and Disease Burden in the United States: A Population-Based Study. Liver Transpl 2019;25:695-705.

4. Sundaram V, Jalan R, Shah P, et al. Acute on Chronic Liver Failure From Nonalcoholic Fatty Liver Disease: A Growing and Aging Cohort With Rising Mortality. Hepatology 2021;73:1932-44.

5. Haldar D, Kern B, Hodson J, et al. Outcomes of liver transplantation for non-alcoholic steatohepatitis: A European Liver Transplant Registry study. J Hepatol 2019;71:313-22.

6. Chalasani N, Younossi Z, Lavine JE, et al. The diagnosis and management of nonalcoholic fatty liver disease: Practice guidance from the American Association for the Study of Liver Diseases. Hepatology 2018;67:328-57.

Cite this article as: Zheng KI, Zheng MH. The uprising of metabolic dysfunction-associated fatty liver disease (MAFLD) in acute-on-chronic liver failure (ACLF). HepatoBiliary Surg Nutr 2021;10(6):857-859. doi: 10.21037/hbsn-21-431
7. Zheng KI, Fan JG, Shi JP, et al. From NAFLD to MAFLD: a "redefining" moment for fatty liver disease. Chin Med J (Engl) 2020;133:2271-3.

8. Zheng KI, Eslam M, George J, et al. When a new definition overhauls perceptions of MAFLD related cirrhosis care. Hepatobiliary Surg Nutr 2020;9:801-4.

9. van der Poorten D, Samer CF, Ramezani-Moghadam $\mathrm{M}$, et al. Hepatic fat loss in advanced nonalcoholic steatohepatitis: are alterations in serum adiponectin the cause? Hepatology 2013;57:2180-8.

10. Thuluvath PJ, Kantsevoy S, Thuluvath AJ, et al. Is cryptogenic cirrhosis different from NASH cirrhosis? J Hepatol 2018;68:519-25.

11. Eslam M, Sarin SK, Wong VWS, et al. The Asian Pacific Association for the Study of the Liver clinical practice guidelines for the diagnosis and management of metabolic associated fatty liver diseases. Hepatol Int 2020;14:889-919.

12. Duseja A, De A, Taneja S, et al. Impact of metabolic risk factors on the severity and outcome of patients with alcohol-associated acute-on-chronic liver failure. Liver Int 2021;41:150-7.

13. Trebicka J, Fernandez J, Papp M, et al. PREDICT identifies precipitating events associated with the clinical course of acutely decompensated cirrhosis. J Hepatol 2021;74:1097-108. 